\title{
Effect of Pb-stress on growth and mineral status of two groundnut (Arachis hypogaea L.) cultivars
}

\author{
Ambekar Nareshkumar, B. V. Krishnappa, T. V. Kirankumar, K. Kiranmai, U. Lokesh, \\ O. Sudhakarbabu, Chinta Sudhakar*
}

Plant Molecular Biology Laboratory, Department of Botany, Sri Krishnadevaraya University, Anantapuramu-515003, Andhra Pradesh, India

Email address:

chintasudhakar@yahoo.com (C. Sudhakar), ambekarnareshrao@gmail.com (A. Nareshkumar)

\section{To cite this article:}

Ambekar Nareshkumar, B. V. Krishnappa, T. V Kirankumar, K. Kiranmai, U. Lokesh, O. Sudhakarbabu, Chinta Sudhakar. Effect of Pb-Stress on Growth and Mineral Status of Two Groundnut (Arachis hypogaea L.) Cultivars. Journal of Plant Sciences. Vol. 2, No. 6, 2014 , pp. $304-310$. doi: $10.11648 /$ j.jps.20140206.17

\begin{abstract}
Heavy metal pollution of air and agricultural soils is one of the most important ecological problems on world scale. Among the heavy metals, lead $(\mathrm{Pb})$ is one of the common environmental pollutants. To investigate $\mathrm{Pb}$ effects on nutrient uptake, two groundnut (Arachis hypogaea L.) cultivars (cultivar K6 and cultivar K9) were grown in pot cultures and stressed with lead nitrate $\left(\mathrm{Pb}\left(\mathrm{NO}_{3}\right)_{2}\right)$ at four concentrations $(100,200,400$ and $800 \mathrm{ppm}) . \mathrm{Pb}$ is accumulated in roots and leaf tissues in dose dependent manner in both groundnut cultivars, which resulted in reduced root and shoot growth and lower uptake of all mineral ions tested. The content of mineral ions such as $\mathrm{Ca}, \mathrm{Na}, \mathrm{Mg}, \mathrm{Co}, \mathrm{Cu}, \mathrm{Ni}, \mathrm{Zn}$ and $\mathrm{Mn}$ reduced in root and leaf tissues of both cultivars due to $\mathrm{Pb}$ stress. But the reduction in mineral ion content was less in cultivar K6 than in cultivar K9. The deficiency of mineral nutrients correlates in a strong decrease in the contents of total chlorophyll, and anthocyanin in both cultivars, but these effects are less pronounced in cultivar K6 than in cultivar K9.
\end{abstract}

Keywords: Pb Stress, Groundnut, Mineral Nutrients

\section{Introduction}

Heavy metal pollution of air and agricultural soils is one of the most important ecological problems on world scale. Contaminant metal can often accumulate in considerable amounts in the plant tissue and exceed the levels that are toxic to man or animal before they produce visible phytotoxic effects [1]. These pollutants persist in the environment for a longer period of time, as they are not easily degraded by soil microorganisms and therefore, can easily be absorbed by plants [2, 3]. The magnitude of $\mathrm{Pb}$ contamination is high relative to that of other heavy metals due to extensive processing of $\mathrm{Pb}$ ore, in addition to wide usage of metal. When taken up by plants, like other metal ions, $\mathrm{Pb}$ interfaces with the physiology and metabolism of the plants by binding to the sulfhydryl groups of various proteins, leading to structure disruption or activity inhibition, and in certain cases causes the displacement of essential elements resulting in deficiency effects $[4,5]$.

Responses of plants to $\mathrm{Pb}$ exposure include decrease in root and shoot growth, plant biomass [6], accelerated leaf senescence [7], inhibition of chlorophyll biosynthesis, inhibition of seed germination, a wide range of adverse effects on growth and metabolism of plants [8], influence the net photosynthetic rate and respiration, and alternate permeability of cell membrane [9]. Many of the observed activates of $\mathrm{Pb}$ appear to be indirect as a result of mineral imbalance within the tissues. Significant changes in nutrient contents as well as in internal ratios of nutrients occur in plants under $\mathrm{Pb}$ toxicity [7]. In most cases $\mathrm{Pb}$ blocks the entry of cations ( $\mathrm{K}, \mathrm{Ca}, \mathrm{Mg}$, $\mathrm{Zn}, \mathrm{Cu}$ and $\mathrm{Fe}$ ) and anions $\left(\mathrm{NO}_{3}^{-}\right)$[9]. $\mathrm{Pb}$ can also alter the activities of the key enzymes of various metabolic pathways such as the photosynthesis, Calvin cycle, nitrogen metabolism, and sugar metabolism [10].

Legumes are reported to be tolerant to several heavy metals [11]. There has been considerable interest in finding legume species which are able to colonize in metal-enriched soils for use in land reclamation or for crop production on marginal soils [1, 12]. Despite the importance of legume crops in maintaining soil fertility and the conflicting reports on the effects of heavy metals, this study was undertaken with groundnut to evaluate the which cultivar is able to resist $\mathrm{Pb}$ stress relatively.

Groundnut, (Arachis hypogaea L.) is a drought tolerant, 
economic and oil seed legume crop. In the present study, we have investigated the effect of $\mathrm{Pb}$ stress on mineral content and its consequences on biomass, chlorophyll and anthocyanin content in two high yielding groundnut cultivars (cultivar K6 and cultivar K9).

\section{Materials and Methods}

\subsection{Plant Growth Conditions and Pb Stress Treatments}

Groundnut (cultivar K6 and K9) seeds were procured from Regional Agricultural Research Station, Acharya NG Ranga Agricultural University (ANGRAU), Kadiri, India, was sown in earthen pots containing air dried red soil and farmyard manure in 3:1 proportion. The pots were kept under natural photoperiod (12-14 hours and temperature $28 \pm 4{ }^{\circ} \mathrm{C}$ ) in the botanical garden and were irrigated once a day with water. After germination, seedlings were thinned to three per pot and maintained for 14 days. 14-day-old plants were subjected to $\mathrm{Pb}$ stress once by adding 0 (control), 100, 200, 400 and 800 ppm of $\mathrm{Pb}$ solution using lead nitrate $\left(\mathrm{Pb}\left(\mathrm{NO}_{3}\right)_{2}\right)$. Both control and treated pots were irrigated daily with tap water. Care was taken while adding water slightly less than field capacity (approximately $300 \mathrm{ml}$ ) to avoid leaching out of solution from treated pots. After 10 days of stress imposition, the plants were uprooted carefully; the leaves and roots were separated, flash frozen in liquid nitrogen and stored at $-80{ }^{\circ} \mathrm{C}$ until further use.

\subsection{Determination of Growth Parameters}

The plants were carefully uprooted from pots and washed thoroughly with running tap water. Plant growth was determined by measuring the length of the root and shoot system. The dry weight (DW) was measured after the shoots and roots were dried at $80^{\circ} \mathrm{C}$ to constant weight. Leaf area was measured by using leaf area meter (Li-Cor, Li3100 USA).

\subsection{Determination of $\mathrm{Pb}$ and Nutrient Elements}

Plants were uprooted and separated into root and leaves, these were thoroughly washed with tap water followed by $0.2 \%$ detergent solution (Tween-20) to remove the waxy / greasy coating on the sample surface. Again, these samples were washed with $0.1 \mathrm{~N} \mathrm{HCl}$ followed by thorough washing with plenty of water and rinsed finally with double distilled water. These samples were dried at $80{ }^{\circ} \mathrm{C}$ in a hot air oven for 24 hours and powdered using mortar and pestle. Oven dried powder sample $(0.5 \mathrm{~g})$ of roots and leaves was taken in a $50 \mathrm{ml}$ boiling test tube and $5 \mathrm{ml}$ of concentrated nitric acid (70\%) was added and incubated at room temperature overnight. The next day, $5 \mathrm{ml}$ of $\mathrm{HNO}_{3}$ and $\mathrm{H}_{2} \mathrm{O}_{2}$ mixture 10: 4 (v: v) was added to it and placed on a mantle (REMI) till all the white fumes evaporated and the thick white residue was left out in flask. It was allowed to cool and volume was made up to $25 \mathrm{ml}$ using ultra pure water. These diluted samples were used for elemental analysis using ICP-OES (Inductively Coupled Plasma - Optical Emission Spectrophotometer) (iCAP-6000 Series, Thermo Scientific, UK). Replications were maintained and their average was used for calculations. The concentrations of elements were expressed as mg or $\mu \mathrm{g} / \mathrm{g}$ dry weight.

\subsection{Determination of Total Chlorophyll Content}

The chlorophyll content was estimated according to the method described by Hiscox and Israelstam [13]. $0.1 \mathrm{~g}$ of leaf bits of both control and stressed plants were placed in a test tube containing $7 \mathrm{ml}$ DMSO (Merck) and chlorophyll was extracted at $65^{\circ} \mathrm{C}$ for $30 \mathrm{~min}$. The liquid was transferred to a $15 \mathrm{ml}$ graduated test tube and the volume was made to $10 \mathrm{ml}$ with DMSO. The absorbance was measured at $645 \mathrm{~nm}, 663$ $\mathrm{nm}$ in a UV-Spectrophotometer (Shimadzu UV-1800) against DMSO as blank. Total chlorophyll content was calculated following the formula used by Arnon [14].

\subsection{Determination of Anthocyanin Content}

Anthocyanins were extracted from $0.5 \mathrm{~g}$ of leaves with $10 \mathrm{ml}$ of mixture of n-propanol: $\mathrm{HCl}: \mathrm{H}_{2} \mathrm{O}(18: 1: 81 \mathrm{v} / \mathrm{v} / \mathrm{v})$. The samples were heated in boiling water bath for $30 \mathrm{~min}$ and they were incubated for $24 \mathrm{~h}$ in the dark at $4{ }^{\circ} \mathrm{C}$. Extracts were filtered and optical density was measured at $\mathrm{A}_{535}$ and $\mathrm{A}_{663}$ anthocyanin contents were calculated according to Lange et al. [15] and were expressed as $A_{535} \mathrm{~g}^{-1}$ fresh weight after correction for Chlorophyll.

\subsection{Data Analysis}

All data were analyzed using the SPSS (Statistical Package for the Social Sciences) version 16.0. Data presented here are mean values and standard deviation $( \pm \mathrm{SD})$. One-way ANOVA was carried out using Post hoc multiple comparison from the Duncan's test at a significance level of $\mathrm{p}<0.05$.

\section{Results and Discussion}

\subsection{Effect of Pb Stress on Plant Growth}

Growth inhibition is a common response to heavy metal stress and is also one of the most important indices of heavy metal tolerance of plants. $\mathrm{Pb}$ is not generally considered to be an essential element for plant growth. The effect of $\mathrm{Pb}$ on seedling growth seems to be different with regards to plant species, cultivars organs and metabolic processes [9]. Groundnut cultivars grown in different concentrations of $\mathrm{Pb}$ exhibited inhibition of both root and shoot growth (table 1). Growth of cultivar K6 was less affected due to $\mathrm{Pb}$ treatments compared with cultivar K9. After exposure to $800 \mathrm{ppm} \mathrm{Pb}$, the root growth was inhibited to $24 \%$ and $46 \%$ in cultivar K6 and $\mathrm{K} 9$ respectively. Whereas, the shoot growth of both cultivars resulted less affected than root and show a decrease about $14.6 \%$ and $38 \%$ in cultivar K6 and K9 respectively.

Morphological changes such as root and shoot growth in response to $\mathrm{Pb}$ treatment has been studied by several investigators. It has been reported that root and shoot growth was reduced in plants $[16,17,18]$ by $\mathrm{Pb}$ stress. The growth of legume plants grown on $\mathrm{Pb}$ ore tailings was reported to be drastically affected [19]. $\mathrm{Pb}$ also inhibited root and shoot 
growth in tobacco [20] and wheat [21]. Similarly, in the present study, the root and shoot growth of cultivar K6 and cultivar $\mathrm{K} 9$ were inhibited by $\mathrm{Pb}$ stress and the reduction was found to be concentration dependent (table 1). However, the percent decrease in root and shoot growth was less in cultivar K6 than in cultivar K9, which indicates the better adaptation of former one to $\mathrm{Pb}$ stress. The reduction in root length and shoot length under $\mathrm{Pb}$ stress may be due to the inhibition in cell elongation process [22] or due to reduced mitotic activity as observed in lupin roots system [23].

\subsection{Effect of Pb on Plant Biomass}

Increased $\mathrm{Pb}$ metal concentration significantly reduced the biomass of two groundnut cultivars (table 1). $\mathrm{Pb}$ induced root and shoot biomass reduction in cultivar K6 was always lower than that of cultivar K9 at all stress treatments. Two cultivars differed from each other in terms of both root and shoot dry mass. At $800 \mathrm{ppm} \mathrm{Pb}$ treatment the reduction in root dry mass was estimated $13.4 \%$ and $29.9 \%$ in cultivar K6 and K9 respectively, compared to the control. Similarly, the shoot dry mass undertake a decrease compared to the control which reached $18.7 \%$ in cultivar $\mathrm{K} 6$ and $47.3 \%$ in cultivar $\mathrm{K} 9$, compared to the control.

Generally, excess concentration of heavy metals in soil results in decreasing plant biomass production. Biomass production has been considered as an index of tolerance level of plants growing on metal enriched soils. Cox and Hutchinson [24] have reported that the dry mass production in non-tolerant plant was significantly negatively correlated with log metal concentration in soil, but the same relationship with tolerant plant was positive. Similarly, in the present study, the dry mass accumulation was much less affected in tolerant cultivar K6 than susceptible cultivar K9 with increasing stress intensity. Concomitantly, Ekmekci et al. [18] reported that the shoot and root ratio (dry mass) was affected by increasing $\mathrm{Pb}$ concentrations in maize. Inhibition of fresh and dry mass accumulation under $\mathrm{Pb}$ stress conditions was also reported in cotton seedlings [25] and in sunflower [26].

\subsection{Effect of Pb on Leaf Area}

The leaf area of cultivar K9 was reduced significantly at all concentrations of $\mathrm{Pb}$ as compared to the control. Whereas, cultivar K6 showed no significant decrease in leaf area due to $\mathrm{Pb}$ treatments (table 1). After exposure to $800 \mathrm{ppm} \mathrm{Pb}$, the decrease in leaf area reached $8.8 \%$ and $34.6 \%$ in cultivar K6 and $\mathrm{K} 9$ respectively compared to their unstressed plants. An increase in the metal supply resulted in inhibition of leaf area in the cotton [25], garden cress [27], and tomato [28, 16]. In contrast, in the present study, $\mathrm{Pb}$ stress didn't induce a significant inhibition of leaf area in cultivar K6.

\subsection{Pb Accumulation in Plant Tissues}

The $\mathrm{Pb}$ content increased in a dose-dependent manner in leaves and roots of two groundnut cultivars (figure 1). At all $\mathrm{Pb}$ stress a level, the $\mathrm{Pb}$ content was lower in leaves and roots of K6 cultivar than that of $\mathrm{K} 9$ cultivar. Furthermore, the $\mathrm{Pb}$ content was relatively higher in the roots than in leaves of both cultivars. At $800 \mathrm{ppm} \mathrm{Pb}$ there was a 4.7-fold increase in $\mathrm{Pb}$ content in leaves and 30.7-fold increase in the roots of cultivar K6 whereas 18-fold increase in leaves and 55-fold increase in roots of cultivar $\mathrm{K} 9$ was observed when compared with their respective controls.

An increase in $\mathrm{Pb}$ content in both cultivars was noticed with increase in the intensity of stress. The increase in the accumulation of $\mathrm{Pb}$ was lesser in cultivar $\mathrm{K} 6$ than in cultivar $\mathrm{K} 9$ under stress conditions. Plants absorb $\mathrm{Pb}$ in its soluble form from soil through roots. Based on comparative studies of metal content in plant parts Baker and Walker [29] suggested that uptake, translocation and accumulation mechanisms differed for various heavy metals and between species and genotypes [30]. It is known that the root system partially defends the above ground parts from $\mathrm{Pb}$ [31], as showed in the present study. Mostly, the plants with highest tolerance take-up the smallest proportion of the total soil-metal and had the lowest shoot metal concentrations [22, 32].
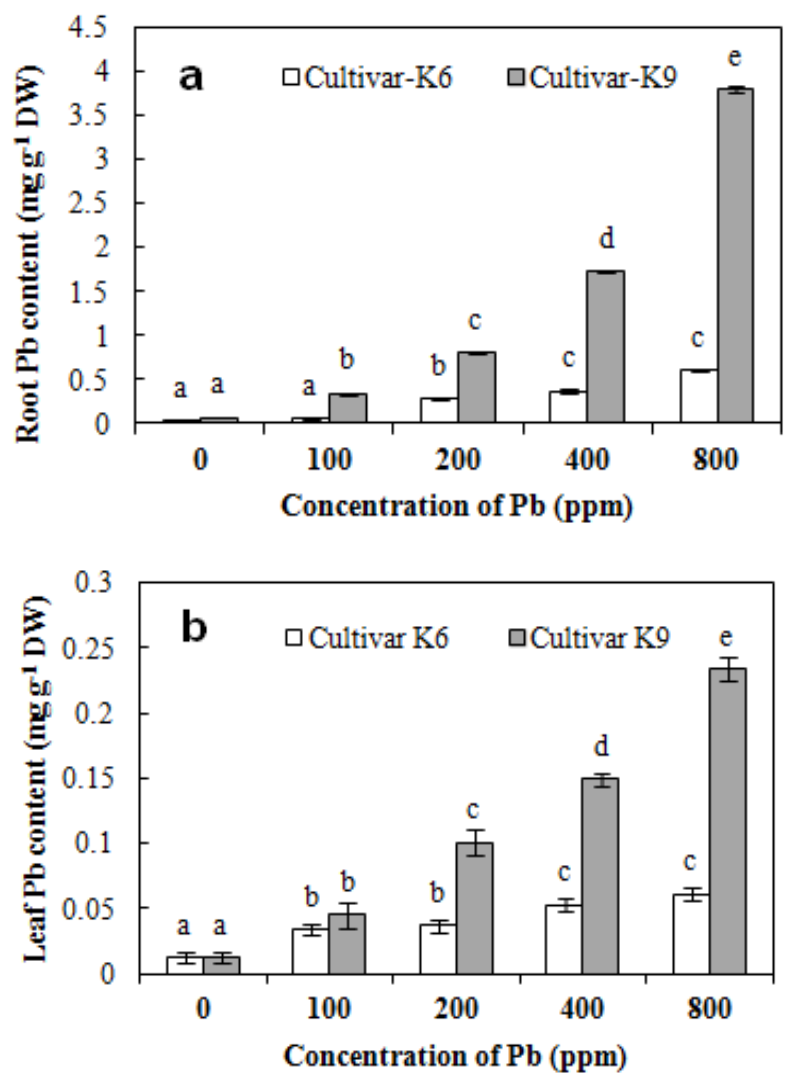

Figure 1. $\mathrm{Pb}$ content in roots $(a)$ and $(b)$ leaves of two groundnut cultivars subjected to $\mathrm{Pb}\left(\mathrm{NO}_{3}\right)_{2}$ stress. The data represent the mean $\pm \mathrm{SD}(n=3)$ of three different experiments and the same letters above the bars are not significantly different at $p<0.05$ (DMR test).

In the same context, in this study although both of cultivars accumulated lower $\mathrm{Pb}$ levels in leaves, tolerant cultivar K6 accumulated still lower proportion of $\mathrm{Pb}$ by restricting the uptake and further translocation of $\mathrm{Pb}$ from roots to leaves. Our study also indicated that the uptake and translocation proportion of $\mathrm{Pb}$ metal vary greatly among these two cultivars and also indicated the retaining ability of the roots to $\mathrm{Pb}$. 


\subsection{Effect of Pb-Stress on Chlorophyll Content}

Chlorosis was associated with reduced leaf chlorophyll content. In wheat seedlings, concentrations of total chlorophyll content decreased significantly in the presence of $\mathrm{Pb}$ in the soil, but this response varied with the concentration of exogenous $\mathrm{Pb}$ [21]. After expose to $800 \mathrm{ppm}$, the reduction was about $22.7 \%$ in cultivar $\mathrm{K} 6$ and $38.5 \%$ in cultivar $\mathrm{K} 9$, compared to respective controls (table 1). The most common symptoms caused by $\mathrm{Pb}$ toxicity were yellowing of the leaves (chlorosis) and leaf curling was noticed at higher concentrations (400 and 800 ppm) especially in cultivar K9.

Total chlorophyll content has been reported to be one of the important indices to assess the tolerance of plants to heavy metals. A reduction in total chlorophyll content during $\mathrm{Pb}$ supply has been noticed in cucumber [32], radish [33] Populus [34] and Vallisneria natans [35]. In the present study, the reduction of total chlorophyll content was less in cultivar K6 suggesting the tolerance of cultivar K6 compared to cultivar K9.

Table 1. Effect of increasing concentrations of $\mathrm{Pb}\left(\mathrm{NO}_{3}\right)_{2}$ on root growth, shoot growth, root dry weight (DW), shoot dry weight (DW), leaf area, total chlorophyll content (TCC) and anthocyanin content of two groundnut cultivars

\begin{tabular}{|c|c|c|c|c|c|c|c|c|}
\hline & $\begin{array}{l}\text { Pb } \\
\text { (ppm) }\end{array}$ & $\begin{array}{l}\text { Root growth } \\
\text { (cm/plant) }\end{array}$ & $\begin{array}{l}\text { Shoot growth } \\
(\mathrm{cm} / \text { plant })\end{array}$ & $\begin{array}{l}\text { Root DW } \\
\left(\mathrm{g}^{-1} / \text { plant }\right) \\
\end{array}$ & $\begin{array}{l}\text { Shoot DW } \\
\left(\mathrm{g}^{-1} \text { plant }\right) \\
\end{array}$ & $\begin{array}{l}\text { Leaf area } \\
\left(\mathrm{cm}^{2}\right)\end{array}$ & $\begin{array}{l}\text { TCC } \\
\left(\mathrm{mg} / \mathrm{g}^{-1} \mathrm{FW}\right)\end{array}$ & $\begin{array}{l}\text { Anthocyanin } \\
\left(\mathrm{mg} / \mathrm{g}^{-1} \text { FW) }\right.\end{array}$ \\
\hline \multirow{5}{*}{$\begin{array}{l}\text { Cultivar } \\
\text { K6 }\end{array}$} & 0 & $\begin{array}{l}19.18 \mathrm{c} \pm 1.97 \\
(100.0)\end{array}$ & $\begin{array}{l}5.74 \mathrm{c} \pm 0.57 \\
(100.0)\end{array}$ & $\begin{array}{l}0.082 \mathrm{~b} \pm 0.004 \\
(100.0)\end{array}$ & $\begin{array}{l}0.325 c \pm 0.017 \\
(100.0)\end{array}$ & $\begin{array}{l}3.60 \mathrm{a} \pm 0.22 \\
(100.0)\end{array}$ & $\begin{array}{l}2.34 c \pm 0.010 \\
(100.0)\end{array}$ & $\begin{array}{l}0.225 \mathrm{a} \pm 0.008 \\
(100.0)\end{array}$ \\
\hline & 100 & $\begin{array}{l}17.02 b \pm 2.24 \\
(88.7)\end{array}$ & $\begin{array}{l}5.55 b c \pm 0.75 \\
(96.6)\end{array}$ & $\begin{array}{l}0.085 b \pm 0.005 \\
(103.6)\end{array}$ & $\begin{array}{l}0.327 c \pm 0.030 \\
(100.6)\end{array}$ & $\begin{array}{l}3.62 \mathrm{a} \pm 0.28 \\
(99.1)\end{array}$ & $\begin{array}{l}2.26 \mathrm{~b} \pm 0.008 \\
(96.1)\end{array}$ & $\begin{array}{l}0.236 \mathrm{a} \pm 0.010 \\
(104.9)\end{array}$ \\
\hline & 200 & $\begin{array}{l}15.66 \mathrm{ab} \pm 1.74 \\
(81.6)\end{array}$ & $\begin{array}{l}5.29 \mathrm{ab} \pm 0.76 \\
(92.1)\end{array}$ & $\begin{array}{l}0.075 \mathrm{a} \pm 0.005 \\
(91.4)\end{array}$ & $\begin{array}{l}0.305 b c \pm 0.018 \\
(93.8)\end{array}$ & $\begin{array}{l}3.47 \mathrm{a} \pm 0.35 \\
(95)\end{array}$ & $\begin{array}{l}2.21 b \pm 0.044 \\
(94.1)\end{array}$ & $\begin{array}{l}0.305 b \pm 0.012 \\
(135.5)\end{array}$ \\
\hline & 400 & $\begin{array}{l}15.06 a \pm 3.85 \\
(78.51)\end{array}$ & $\begin{array}{l}4.94 a \pm 0.74 \\
(86.06)\end{array}$ & $\begin{array}{l}0.075 \mathrm{a} \pm 0.005 \\
(87.8)\end{array}$ & $\begin{array}{l}0.297 \mathrm{~b} \pm 0.017 \\
(91.4)\end{array}$ & $\begin{array}{l}3.46 \mathrm{a} \pm 0.35 \\
(94.8)\end{array}$ & $\begin{array}{l}2.03 b \pm 0.047 \\
(86.6)\end{array}$ & $\begin{array}{l}0.417 c \pm 0.041 \\
(185.3)\end{array}$ \\
\hline & 800 & $\begin{array}{l}14.57 \mathrm{a} \pm 2.62 \\
(75.9)\end{array}$ & $\begin{array}{l}4.90 \mathrm{a} \pm 0.73 \\
(85.3)\end{array}$ & $\begin{array}{l}0.071 \mathrm{a} \pm 0.006 \\
(86.6)\end{array}$ & $\begin{array}{l}0.264 a \pm 0.036 \\
(81.2)\end{array}$ & $\begin{array}{l}3.33 \mathrm{a} \pm 0.47 \\
(91.2)\end{array}$ & $\begin{array}{l}1.93 \mathrm{a} \pm 0.041 \\
(82.4)\end{array}$ & $\begin{array}{l}0.826 \mathrm{~d} \pm 0.025 \\
(367.1)\end{array}$ \\
\hline \multirow{5}{*}{$\begin{array}{l}\text { Cultivar } \\
\text { K9 }\end{array}$} & 0 & $\begin{array}{l}23.37 d \pm 3.96 \\
(100.0)\end{array}$ & $\begin{array}{l}1.07 \mathrm{~d} \pm 0.08 \\
(100.0)\end{array}$ & $\begin{array}{l}0.077 \mathrm{~d} \pm 0.004 \\
(100.0)\end{array}$ & $\begin{array}{l}0.338 \mathrm{e} \pm 0.030 \\
(100.0)\end{array}$ & $\begin{array}{l}2.66 c \pm 0.29 \\
(100.0)\end{array}$ & $\begin{array}{l}2.44 d \pm 0.056 \\
(100.0)\end{array}$ & $\begin{array}{l}0.600 \mathrm{~d} \pm 0.030 \\
(100.0)\end{array}$ \\
\hline & 100 & $\begin{array}{l}18.19 \mathrm{c} \pm 3.38 \\
(77.8)\end{array}$ & $\begin{array}{l}5.37 d \pm 0.72 \\
(82.8)\end{array}$ & $\begin{array}{l}0.068 \mathrm{c} \pm 0.005 \\
(88.3)\end{array}$ & $\begin{array}{l}0.312 d \pm 0.042 \\
(92.3)\end{array}$ & $\begin{array}{l}2.53 c \pm 0.32 \\
(95.1)\end{array}$ & $\begin{array}{l}2.30 \mathrm{~d} \pm 0.029 \\
(94.6)\end{array}$ & $\begin{array}{l}0.648 \mathrm{e} \pm 0.030 \\
(108)\end{array}$ \\
\hline & 200 & $\begin{array}{l}16.62 b c \pm 2.83 \\
(71.1)\end{array}$ & $\begin{array}{l}4.45 c \pm 0.51 \\
(80.03)\end{array}$ & $\begin{array}{l}0.061 b \pm 0.004 \\
(79.2)\end{array}$ & $\begin{array}{l}0.268 \mathrm{c} \pm 0.020 \\
(79.2)\end{array}$ & $\begin{array}{l}2.08 b \pm 0.17 \\
(78.2)\end{array}$ & $\begin{array}{l}2.12 \mathrm{c} \pm 0.028 \\
(87.2)\end{array}$ & $\begin{array}{l}0.560 \mathrm{c} \pm 0.017 \\
(93.3)\end{array}$ \\
\hline & 400 & $\begin{array}{l}15.89 \mathrm{~b} \pm 3.25 \\
(67.9)\end{array}$ & $\begin{array}{l}3.94 b \pm 0.37 \\
(70.86)\end{array}$ & $\begin{array}{l}0.057 \mathrm{ab} \pm 0.005 \\
(74)\end{array}$ & $\begin{array}{l}0.243 b \pm 0.018 \\
(71.9)\end{array}$ & $\begin{array}{l}1.96 b \pm 0.16 \\
(73.7)\end{array}$ & $\begin{array}{l}1.84 \mathrm{~b} \pm 0.023 \\
(75.5)\end{array}$ & $\begin{array}{l}0.469 \mathrm{~b} \pm 0.019 \\
(78.1)\end{array}$ \\
\hline & 800 & $\begin{array}{l}12.66 \mathrm{a} \pm 3.06 \\
(54.1)\end{array}$ & $\begin{array}{l}3.45 \mathrm{a} \pm 0.64 \\
(62.05)\end{array}$ & $\begin{array}{l}0.054 \mathrm{a} \pm 0.004 \\
(70)\end{array}$ & $\begin{array}{l}0.178 \mathrm{a} \pm 0.014 \\
(52.6)\end{array}$ & $\begin{array}{l}1.74 \mathrm{e} \pm 0.16 \\
(65.4)\end{array}$ & $\begin{array}{l}1.55 \mathrm{a} \pm 0.160 \\
(63.8)\end{array}$ & $\begin{array}{l}0.317 \mathrm{e} \pm 0.015 \\
(52.8)\end{array}$ \\
\hline
\end{tabular}

(Percentages to control in parenthesis)

The data represent the mean $\pm \mathrm{SD}(\mathrm{n}=10)$ of three different experiments and the same letters after averages are not significantly different at $\mathrm{p}<0.05$ (DMR test).

Table 2. Effect of $\mathrm{Pb}\left(\mathrm{NO}_{3}\right)_{2}$ on leaf elemental profile of two groundnut cultivars.

\begin{tabular}{|c|c|c|c|c|c|c|c|c|c|c|}
\hline & $\begin{array}{l}\mathbf{P b} \\
(\mathbf{p p m})\end{array}$ & $\begin{array}{l}\mathrm{Ca} \\
\left(\mathrm{mg} / \mathrm{g}^{-1} \mathrm{DW}\right)\end{array}$ & $\begin{array}{l}\text { Mg } \\
\left(\mathrm{mg} / \mathrm{g}^{-1} \mathrm{DW}\right)\end{array}$ & $\begin{array}{l}\mathrm{Na} \\
\left(\mathrm{mg} / \mathrm{g}^{-1} \mathrm{DW}\right)\end{array}$ & $\begin{array}{l}\mathrm{Cu} \\
\left(\mu \mathrm{g} / \mathrm{g}^{-1} \mathrm{DW}\right)\end{array}$ & $\begin{array}{l}\text { Co } \\
\left(\mu \mathrm{g} / \mathrm{g}^{-1} \mathrm{DW}\right)\end{array}$ & $\begin{array}{l}\text { Fe } \\
\left(\mu g / g^{-1} D W\right)\end{array}$ & $\begin{array}{l}\text { Mn } \\
\left(\mu \mathrm{g} / \mathrm{g}^{-1} \mathrm{DW}\right)\end{array}$ & $\begin{array}{l}\mathrm{Ni} \\
\left(\mu \mathrm{g} / \mathrm{g}^{-1} \mathrm{DW}\right)\end{array}$ & $\begin{array}{l}\mathrm{Zn} \\
\left(\mu \mathrm{g} / \mathrm{g}^{-1} \mathrm{DW}\right)\end{array}$ \\
\hline \multirow{3}{*}{ 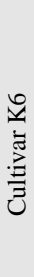 } & 0 & $\begin{array}{l}25.93 e \pm 1.35 \\
(100.0)\end{array}$ & $\begin{array}{l}10.25 d \pm 0.06 \\
(100.0)\end{array}$ & $\begin{array}{l}0.108 d \pm 0.015 \\
(100.0)\end{array}$ & $\begin{array}{l}18.02 \mathrm{~d} \pm 0.18 \\
(100.0)\end{array}$ & $\begin{array}{l}4.16 b \pm 0.09 \\
(100.0)\end{array}$ & $\begin{array}{l}0.150 \mathrm{c} \pm 0.013 \\
(100.0)\end{array}$ & $\begin{array}{l}53.20 \mathrm{~d} \pm 2.35 \\
(100.0)\end{array}$ & $\begin{array}{l}8.16 \mathrm{~d} \pm 0.08 \\
(100.0)\end{array}$ & $\begin{array}{l}0.264 \mathrm{e} \pm 0.011 \\
(100.0)\end{array}$ \\
\hline & 100 & $\begin{array}{l}25.50 \mathrm{~d} \pm 1.05 \\
(98.3)\end{array}$ & $\begin{array}{l}9.56 c \pm 0.07 \\
(93.2)\end{array}$ & $\begin{array}{l}0.107 \mathrm{c} \pm 0.012 \\
(99.0)\end{array}$ & $\begin{array}{l}14.18 \mathrm{c} \pm 0.17 \\
(79.7)\end{array}$ & $\begin{array}{l}4.16 \mathrm{ab} \pm 0.03 \\
(100.0)\end{array}$ & $\begin{array}{l}0.135 b \pm 0.012 \\
(90.6)\end{array}$ & $\begin{array}{l}50.82 c \pm 3.72 \\
(95.5)\end{array}$ & $\begin{array}{l}8.08 c \pm 0.08 \\
(99.0)\end{array}$ & $\begin{array}{l}0.251 \mathrm{~d} \pm 0.017 \\
(95.0)\end{array}$ \\
\hline & 200 & $\begin{array}{l}17.90 \mathrm{c} \pm 1.23 \\
(69.0)\end{array}$ & $\begin{array}{l}9.13 b \pm 0.10 \\
(89.0)\end{array}$ & $\begin{array}{l}0.103 b \pm 0.011 \\
(95.4)\end{array}$ & $\begin{array}{l}12.56 b \pm 0.16 \\
(78.7)\end{array}$ & $\begin{array}{l}4.16 \mathrm{ab} \pm 0.03 \\
(100.0)\end{array}$ & $\begin{array}{l}0.134 b \pm 0.009 \\
(90.3)\end{array}$ & $\begin{array}{l}50.29 b c \pm 2.62 \\
(94.5)\end{array}$ & $\begin{array}{l}7.97 b c \pm 0.09 \\
(97.6)\end{array}$ & $\begin{array}{l}0.248 c \pm 0.019 \\
(93.9)\end{array}$ \\
\hline \multirow{5}{*}{ 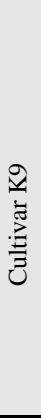 } & 800 & $\begin{array}{l}15.73 \mathrm{a} \pm 1.07 \\
(60.6)\end{array}$ & $\begin{array}{l}8.25 a \pm 0.10 \\
(80.4)\end{array}$ & $\begin{array}{l}0.094 \mathrm{a} \pm 0.009 \\
(87.0)\end{array}$ & $\begin{array}{l}11.44 a \pm 0.16 \\
(63.5)\end{array}$ & $\begin{array}{l}4.10 \mathrm{a} \pm 0.05 \\
(98.5)\end{array}$ & $\begin{array}{l}0.105 a \pm 0.007 \\
(70.7)\end{array}$ & $\begin{array}{l}48.02 \mathrm{a} \pm 3.77 \\
(90.2)\end{array}$ & $\begin{array}{l}7.70 \mathrm{a} \pm 0.13 \\
(94.4)\end{array}$ & $\begin{array}{l}0.230 \mathrm{a} \pm 0.014 \\
(87.1)\end{array}$ \\
\hline & 0 & $\begin{array}{l}26.33 \mathrm{e} \pm 1.12 \\
(100.0)\end{array}$ & $\begin{array}{l}12.37 \mathrm{e} \pm 0.05 \\
(100.0)\end{array}$ & $\begin{array}{l}0.113 e \pm 0.005 \\
(100.0)\end{array}$ & $\begin{array}{l}67.81 \mathrm{~d} \pm 0.11 \\
(100.0)\end{array}$ & $\begin{array}{l}4.16 b \pm 0.05 \\
(100.0)\end{array}$ & $\begin{array}{l}0.191 d \pm 0.009 \\
(100.0)\end{array}$ & $\begin{array}{l}59.60 d \pm 4.63 \\
(100.0)\end{array}$ & $\begin{array}{l}9.38 \mathrm{~d} \pm 0.05 \\
(100.0)\end{array}$ & $\begin{array}{l}0.266 \mathrm{~d} \pm 0.011 \\
(100.0)\end{array}$ \\
\hline & 100 & $\begin{array}{l}22.07 d \pm 1.01 \\
(83.8)\end{array}$ & $\begin{array}{l}10.17 d \pm 0.09 \\
(82.2)\end{array}$ & $\begin{array}{l}0.104 d \pm 0.008 \\
(92.0)\end{array}$ & $\begin{array}{l}28.61 \mathrm{c} \pm 0.17 \\
(42.2)\end{array}$ & $\begin{array}{l}4.13 b \pm 0.02 \\
(99.3)\end{array}$ & $\begin{array}{l}0.151 \mathrm{c} \pm 0.015 \\
(79.1)\end{array}$ & $\begin{array}{l}58.13 \mathrm{~cd} \pm 2.58 \\
(93.0)\end{array}$ & $\begin{array}{l}8.16 c \pm 0.08 \\
(86.9)\end{array}$ & $\begin{array}{l}0.255 \mathrm{c} \pm 0.011 \\
(95.8)\end{array}$ \\
\hline & 400 & $\begin{array}{l}15.72 b \pm 1.07 \\
(59.3)\end{array}$ & $\begin{array}{l}8.38 b \pm 0.05 \\
(67.7)\end{array}$ & $\begin{array}{l}0.088 b \pm 0.004 \\
(78.0)\end{array}$ & $\begin{array}{l}18.66 b \pm 0.19 \\
(27.5)\end{array}$ & $\begin{array}{l}4.08 \mathrm{ab} \pm 0.02 \\
(98.0)\end{array}$ & $\begin{array}{l}0.144 b \pm 0.006 \\
(75.4)\end{array}$ & $\begin{array}{l}53.70 b \pm 3.82 \\
(90.5)\end{array}$ & $\begin{array}{l}7.86 b \pm 0.05 \\
(83.8)\end{array}$ & $\begin{array}{l}0.238 b \pm 0.011 \\
(89.4)\end{array}$ \\
\hline & 800 & $\begin{array}{l}7.72 a \pm 1.13 \\
(29.7)\end{array}$ & $\begin{array}{l}8.20 \mathrm{a} \pm 0.06 \\
(66.3)\end{array}$ & $\begin{array}{l}0.084 a \pm 0.004 \\
(47.1)\end{array}$ & $\begin{array}{l}14.10 \mathrm{a} \pm 0.32 \\
(20.7)\end{array}$ & $\begin{array}{l}4.08 \mathrm{a} \pm 0.01 \\
(98.0)\end{array}$ & $\begin{array}{l}0.131 \mathrm{a} \pm 0.002 \\
(68.4)\end{array}$ & $\begin{array}{l}51.04 \mathrm{a} \pm 4.37 \\
(82.2)\end{array}$ & $\begin{array}{l}7.65 \mathrm{a} \pm 0.05 \\
(81.5)\end{array}$ & $\begin{array}{l}0.227 \mathrm{a} \pm 0.015 \\
(85.3)\end{array}$ \\
\hline
\end{tabular}

(Percentages to control in parenthesis)

The data represent the mean $\pm \mathrm{SD}(\mathrm{n}=3$ ) of three different experiments and the same letters after average values are not significantly different at $\mathrm{p}<0.05$ (DMR test). 
Table 3. Effect of $\mathrm{Pb}\left(\mathrm{NO}_{3}\right)_{2}$ on root elemental profile of two groundnut cultivars.

\begin{tabular}{|c|c|c|c|c|c|c|c|c|c|c|}
\hline & $\begin{array}{l}\text { Pb } \\
(\mathbf{p p m})\end{array}$ & $\begin{array}{l}\text { Ca } \\
\left(\mathrm{mg} / \mathrm{g}^{-1} \mathrm{DW}\right)\end{array}$ & $\begin{array}{l}\mathrm{Mg} \\
\left(\mathrm{mg} / \mathrm{g}^{-1} \mathrm{DW}\right)\end{array}$ & $\begin{array}{l}\mathrm{Na} \\
\left(\mathrm{mg} / \mathrm{g}^{-1} \mathrm{DW}\right)\end{array}$ & $\begin{array}{l}\mathrm{Cu} \\
\left(\mu \mathrm{g} / \mathrm{g}^{-1} \mathrm{DW}\right)\end{array}$ & $\begin{array}{l}\text { Co } \\
\left(\mu \mathrm{g} / \mathrm{g}^{-1} \mathrm{DW}\right)\end{array}$ & $\begin{array}{l}\mathrm{Fe} \\
\left(\mu \mathrm{g} / \mathrm{g}^{-1} \mathrm{DW}\right)\end{array}$ & $\begin{array}{l}\text { Mn } \\
\left(\mu \mathrm{g} / \mathrm{g}^{-1} \mathrm{DW}\right)\end{array}$ & $\begin{array}{l}\text { Ni } \\
\left(\mu \mathrm{g} / \mathrm{g}^{-1} \mathrm{DW}\right)\end{array}$ & $\begin{array}{l}Z n \\
\left(\mu g / g^{-1} D W\right)\end{array}$ \\
\hline \multirow{4}{*}{ 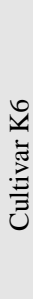 } & 0 & $\begin{array}{l}21.61 \mathrm{e} \pm 0.41 \\
(100.0)\end{array}$ & $\begin{array}{l}13.89 \mathrm{~d} \pm 0.28 \\
(100.0)\end{array}$ & $\begin{array}{l}0.45 a \pm 0.011 \\
(100.0)\end{array}$ & $\begin{array}{l}33.78 \mathrm{~d} \pm 1.41 \\
(100.0)\end{array}$ & $\begin{array}{l}2.24 b \pm 0.081 \\
(100.0)\end{array}$ & $\begin{array}{l}1.50 \mathrm{c} \pm 0.015 \\
(100.0)\end{array}$ & $\begin{array}{l}42.56 \mathrm{c} \pm 0.24 \\
(100.0)\end{array}$ & $\begin{array}{l}10.01 \mathrm{~d} \pm 0.08 \\
(100.0)\end{array}$ & $\begin{array}{l}0.156 \mathrm{e} \pm 0.007 \\
(100.0)\end{array}$ \\
\hline & 100 & $\begin{array}{l}19.70 \mathrm{~d} \pm 0.63 \\
(91.2)\end{array}$ & $\begin{array}{l}13.26 \mathrm{c} \pm 0.17 \\
(99.5)\end{array}$ & $\begin{array}{l}0.44 \mathrm{c} \pm 0.014 \\
(99.5)\end{array}$ & $\begin{array}{l}33.78 c \pm 1.53 \\
(100.0)\end{array}$ & $\begin{array}{l}2.16 \mathrm{ab} \pm 0.085 \\
(96.4)\end{array}$ & $\begin{array}{l}1.44 \mathrm{~b} \pm 0.184 \\
(96.0)\end{array}$ & $\begin{array}{l}41.92 \mathrm{c} \pm 0.41 \\
(98.5)\end{array}$ & $\begin{array}{l}9.78 \mathrm{c} \pm 0.12 \\
(97.8)\end{array}$ & $\begin{array}{l}0.137 \mathrm{~d} \pm 0.009 \\
(87.8)\end{array}$ \\
\hline & 200 & $\begin{array}{l}18.66 \mathrm{c} \pm 0.41 \\
(86.3)\end{array}$ & $\begin{array}{l}11.67 b \pm 0.36 \\
(97.3)\end{array}$ & $\begin{array}{l}0.43 b \pm 0.015 \\
(97.3)\end{array}$ & $\begin{array}{l}31.06 \mathrm{~b} \pm 1.80 \\
(97.9)\end{array}$ & $\begin{array}{l}1.81 \mathrm{ab} \pm 0.122 \\
(80.8)\end{array}$ & $\begin{array}{l}1.36 \mathrm{~b} \pm 0.039 \\
(90.2)\end{array}$ & $\begin{array}{l}37.33 b c \pm 0.69 \\
(87.7)\end{array}$ & $\begin{array}{l}9.01 b c \pm 0.44 \\
(90.1)\end{array}$ & $\begin{array}{l}0.132 c \pm 0.008 \\
(84.6)\end{array}$ \\
\hline & 400 & $\begin{array}{l}17.60 \mathrm{a} \pm 0.80 \\
(81.5)\end{array}$ & $\begin{array}{l}11.72 b \pm 0.11 \\
(95.8)\end{array}$ & $\begin{array}{l}0.43 b \pm 0.013 \\
(95.7)\end{array}$ & $\begin{array}{l}30.93 a \pm 1.36 \\
(91.5)\end{array}$ & $\begin{array}{l}1.78 \mathrm{ab} \pm 0.08 \\
(79.5)\end{array}$ & $\begin{array}{l}1.26 \mathrm{~b} \pm 0.021 \\
(83.2)\end{array}$ & $\begin{array}{l}34.16 \mathrm{~b} \pm 0.64 \\
(80.2)\end{array}$ & $\begin{array}{l}8.08 \mathrm{c} \pm 0.08 \\
(80.8)\end{array}$ & $\begin{array}{l}0.129 \mathrm{~b} \pm 0.012 \\
(82.7)\end{array}$ \\
\hline \multirow{6}{*}{ 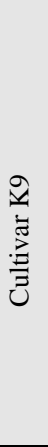 } & 800 & $\begin{array}{l}17.05 b \pm 0.45 \\
(83.0)\end{array}$ & $\begin{array}{l}11.63 a \pm 0.08 \\
(95.3)\end{array}$ & $\begin{array}{l}0.42 \mathrm{a} \pm 0.015 \\
(95.3)\end{array}$ & $\begin{array}{l}29.52 \mathrm{a} \pm 1.26 \\
(87.3)\end{array}$ & $\begin{array}{l}1.62 \mathrm{a} \pm 0.046 \\
(72.3)\end{array}$ & $\begin{array}{l}1.24 \mathrm{~b} \pm 0.003 \\
(77.5)\end{array}$ & $\begin{array}{l}33.01 \mathrm{a} \pm 0.44 \\
(77.5)\end{array}$ & $\begin{array}{l}7.97 \mathrm{a} \pm 0.09 \\
(79.7)\end{array}$ & $\begin{array}{l}0.124 \mathrm{a} \pm 0.008 \\
(79.5)\end{array}$ \\
\hline & 0 & $\begin{array}{l}23.46 a \pm 0.60 \\
(100.0)\end{array}$ & $\begin{array}{l}16.52 \mathrm{e} \pm 0.26 \\
(100.0)\end{array}$ & $\begin{array}{l}0.51 \mathrm{e} \pm 0.013 \\
(100.0)\end{array}$ & $\begin{array}{l}34.66 \mathrm{~d} \pm 2.38 \\
(100.0)\end{array}$ & $\begin{array}{l}3.49 \mathrm{~b} \pm 0.046 \\
(100.0)\end{array}$ & $\begin{array}{l}2.38 \mathrm{a} \pm 0.015 \\
(100.0)\end{array}$ & $\begin{array}{l}57.12 \mathrm{~d} \pm 0.49 \\
(100.0)\end{array}$ & $\begin{array}{l}10.67 d \pm 0.16 \\
(100.0)\end{array}$ & $\begin{array}{l}0.192 \mathrm{~d} \pm 0.009 \\
(100.0)\end{array}$ \\
\hline & 100 & $\begin{array}{l}20.73 c \pm 0.33 \\
(88.3)\end{array}$ & $\begin{array}{l}14.84 d \pm 0.27 \\
(89.5)\end{array}$ & $\begin{array}{l}0.46 \mathrm{~d} \pm 0.012 \\
(89.5)\end{array}$ & $\begin{array}{l}33.73 \mathrm{c} \pm 1.88 \\
(97.3)\end{array}$ & $\begin{array}{l}2.61 \mathrm{ab} \pm 0.046 \\
(74.8)\end{array}$ & $\begin{array}{l}1.97 \mathrm{c} \pm 0.011 \\
(95.2)\end{array}$ & $\begin{array}{l}54.40 \mathrm{~cd} \pm 0.08 \\
(95.2)\end{array}$ & $\begin{array}{l}10.05 \mathrm{c} \pm 0.17 \\
(94.1)\end{array}$ & $\begin{array}{l}0.173 c \pm 0.008 \\
(90.1)\end{array}$ \\
\hline & 200 & $\begin{array}{l}19.45 b \pm 0.43 \\
(83.0)\end{array}$ & $\begin{array}{l}14.51 \mathrm{c} \pm 0.10 \\
(87.8)\end{array}$ & $\begin{array}{l}0.36 \mathrm{c} \pm 0.013 \\
(70.0)\end{array}$ & $\begin{array}{l}33.20 \mathrm{~b} \pm 1.67 \\
(95.8)\end{array}$ & $\begin{array}{l}2.58 \mathrm{ab} \pm 0.046 \\
(73.9)\end{array}$ & $\begin{array}{l}1.8 \mathrm{bc} \pm 0.044 \\
(92.2)\end{array}$ & $\begin{array}{l}52.66 \mathrm{c} \pm 0.76 \\
(92.1)\end{array}$ & $\begin{array}{l}8.93 b \pm 0.20 \\
(83.7)\end{array}$ & $\begin{array}{l}0.172 b \pm 0.006 \\
(89.6)\end{array}$ \\
\hline & 400 & $\begin{array}{l}19.74 \mathrm{c} \pm 0.75 \\
(84.2)\end{array}$ & $\begin{array}{l}13.95 b \pm 0.19 \\
(84.5)\end{array}$ & $\begin{array}{l}0.35 b \pm 0.018 \\
(68.3)\end{array}$ & $\begin{array}{l}31.28 b \pm 1.66 \\
(90.2)\end{array}$ & $\begin{array}{l}1.84 \mathrm{~b} \pm 0.081 \\
(52.7)\end{array}$ & $\begin{array}{l}1.60 \mathrm{c} \pm 0.022 \\
(73.1)\end{array}$ & $\begin{array}{l}41.78 b \pm 0.56 \\
(73.1)\end{array}$ & $\begin{array}{l}7.81 b \pm 0.28 \\
(83.7)\end{array}$ & $\begin{array}{l}0.158 \mathrm{~b} \pm 0.006 \\
(82.3)\end{array}$ \\
\hline & 800 & $\begin{array}{l}18.01 \mathrm{a} \pm 0.53 \\
(76.7)\end{array}$ & $\begin{array}{l}12.11 \mathrm{a} \pm 0.21 \\
(73.3)\end{array}$ & $\begin{array}{l}0.32 \mathrm{a} \pm 0.015 \\
(63.6)\end{array}$ & $\begin{array}{l}28.26 a \pm 1.38 \\
(81.5)\end{array}$ & $\begin{array}{l}1.36 \mathrm{~b} \pm 0.081 \\
(38.9)\end{array}$ & $\begin{array}{l}1.48 \mathrm{a} \pm 0.030 \\
(60.1)\end{array}$ & $\begin{array}{l}37.45 \mathrm{a} \pm 1.97 \\
(65.4)\end{array}$ & $\begin{array}{l}6.66 a \pm 0.17 \\
(66.6)\end{array}$ & $\begin{array}{l}0.101 \mathrm{a} \pm 0.008 \\
(52.6)\end{array}$ \\
\hline
\end{tabular}

(Figures in parenthesis are per cent values)

The data represent the mean \pm SD $(n=3)$ of three different experiments and the same letters after averages values are not significantly different at $\mathrm{p}<0.05$ (DMR test).

\subsection{Effect of Pb-Stress on Anthocyanin Content}

Kumar et al., [36] suggested that in plants, the synthesis of anthocyanins makes it an effective strategy against ROS generation due to $\mathrm{Pb}$ stress, but interestingly lower concentrations of $\mathrm{Pb}$ stimulated synthesis of anthocyanins more than higher concentrations of $\mathrm{Pb}$. Anthocyanin concentration increase in leaves of both the cultivars exposed to increasing $\mathrm{Pb}$ concentrations. However, this increase was always higher in cultivar K6 than in cultivar K9. This indicates that there is a strong correlation between the level of anthocyanins and the presence of heavy metals in the growth medium (table 1).

\subsection{Effect of Pb on Mineral Contents}

Table 2, 3 showed that high $\mathrm{Pb}$ concentrations in the soil cause a reduction of most macro-elements $(\mathrm{Ca}, \mathrm{Mg}$ and $\mathrm{Na}$ ). microelements $(\mathrm{Cu}, \mathrm{Fe}, \mathrm{Mn}, \mathrm{Ni}$ and $\mathrm{Zn})$ are also reduced in both the cultivars. By contrast, Co concentrations are not significantly changed in leaves of both the cultivars. Thus, we can conclude that the uptake of all the measured nutrients is reduced by $\mathrm{Pb}$ treatment. In order to better visualize the effects of $\mathrm{Pb}$, the data have been expressed as the percentage change relative to dry weight and for total amounts per plant. It is known that $\mathrm{Pb}$ physically blocks the access of many ions to their absorption sites on the roots [37], thus inhibiting their uptake. However, the very large reductions of ionic content observed in the present study can hardly result from just an inhibition of ion uptake, and they probably also result from additional ion leakage from the plants. In all cases, cultivar K6 appears less affected than cultivar $\mathrm{K} 9$ by $\mathrm{Pb}$ treatment. $\mathrm{Ca}$ and $\mathrm{Cu}$ ion concentration seems very sensitive to $\mathrm{Pb}$ treatments, and the lowest $\mathrm{Pb}$ concentration already has very large effects.
This observation also applies to $\mathrm{Ca}, \mathrm{Mg}, \mathrm{Na}, \mathrm{Co}, \mathrm{Mn}, \mathrm{Ni}$ and $\mathrm{Zn}$ in roots in the case of cultivar $\mathrm{K} 9$, whereas in cultivar K6 these levels are only slightly disturbed by $400 \mathrm{ppm} \mathrm{Pb}$.

\section{Conclusions}

Our results demonstrated that $\mathrm{Pb}$ treatment even at low concentrations induces large disturbances in ion uptake by plants, which results in serious metabolic changes (e.g., in photosynthetic capacity) (unpublished data) and finally in a strong inhibition of cultivar K6 as a result of the deleterious effects of $\mathrm{Pb}$ on plant growth. Two groundnut cultivars exhibit different sensitiveness to $\mathrm{Pb}$ treatment, and cultivar $\mathrm{K} 6$ appears more resistant. Future experiments will be aimed at searching for the mechanisms responsible for the improved protection of cultivar K6 against the deleterious effects of $\mathrm{Pb}$.

\section{Acknowledgements}

We acknowledge the DST (SR/SO/PS/001/2011 and CSIR-(38/1305/11/EMR-II), GoI, New Delhi for financial assistance in the form of research grants to Chinta Sudhakar. We thank the DST-PURSE facility, Sri Venkateswara University, Tirupati for ICP-OES analysis.

\section{References}

[1] A. M. Reddy, S. G. Kumar, G. Jyothsnakumar, S. Thimmanaik, and C. Sudhakar, Lead induced changes in antioxidant metabolism of horsegram (Macrotyloma uniflorum (Lam.) Verdc.) and bengalgram (Cicer arietinum L.). Chemosphere, 60 (2005) 97-104. 
[2] M. Raghunath, E. A. Putnam, T. Ritty, D. Hamstra, E. S. Park, M. Tschödrich-Rotter, R. Peters, A. Rehemtulla, and D. M. Milewicz, Carboxy-terminal conversion of profibrillin to fibrillin at a basic site by PACE/furin-like activity required for incorporation in the matrix. Journal of Cell Science, 112 (1999) 1093-1100.

[3] S. Gallego, M. Benavides, and M. Tomaro, Involvement of an antioxidant defense system in the adaptive response to heavy metal ions in Helianthus annuus L. cells. Plant Growth Regulation, 36 (2002) 267-273.

[4] F. V. Assche, and H. Clijsters, Effects of metal on enzyme activity in plants. Plant Cell \& Environment, 13 (1990) 195-206.

[5] C. M. Luna, C. A. Gonzalez, and V. S. Trippi, Oxidative damage caused by an excess of copper in oat leaves. Plant \& Cell Physiology, 35 (1994) 11-15.

[6] A. Fargasova, Phytotoxic effects of $\mathrm{Cd}, \mathrm{Zn}, \mathrm{Pb}, \mathrm{Cu}$ and $\mathrm{Fe}$ on Sinapis alba L. seedling and their accumulation in roots and shoots. Biologia Plantarum, 44 (2001) 471-473.

[7] M. Moustakas, T. Lanaras, L. Symeonidis, and S. Karataglis. Growth and some photosynthetic characteristics of field grown Avena sativa under copper and lead stress. Photosynthetica, 30 (1994) 389-396.

[8] P. Sharma, and R. S. Dubey, Ascorbate peroxidase from rice seedlings; properties of enzyme isoforms, effects of stress and protective roles of osmolytes. Plant Science, 167 (2004) 541-550.

[9] A. Kabata-Pendias, and H. Pendias, Trace elements in soils and plants. 1992, $2^{\text {nd }}$ edn. CRC Press, Boca Raton, London.

[10] J. Varma, and N. K. Dubey, Efficacy of essential oils of Caesulia axillaris and Mentha arvensis against some storage pests causing biodeterioration of food commodities. International Journal of Food Microbiology, 68 (2001) 207-210.

[11] J. A. C. Verkleij, A. Golan-Goldhirsh, D. M. Antosiewisz, J. P. Schwitzguébel, and P. Schröder, Dualities in plant tolerance to pollutants and their uptake and translocation to the upper plant parts. Environmental and Experimental Botany, 1 (2009) 10-22.

[12] H. H. Zahran, Rhizobium-Legume symbiosis and nitrogen fixation under severe conditions and in an arid climate. In Microbiology and Molecular Biology Reviews, American society for microbiology, (1999) 63, pp. 968-989.

[13] J. D. Hiscox, and G. F. Israelstam, A method for extraction of chlorophyll from leaf tissues without maceration. Canadian Journal of Botany, 57 (1979) 1332-1334.

[14] D. I. Arnon, Copper enzymes in isolated chloroplasts. Polyphenol oxidase in Beta vulgaris. Plant Physiology, 24 (1949) 1-15.

[15] H. Lange, W. Shropshire, and H. Mohr, An Analysis of Phytochrome-mediated Anthocyanin Synthesis. Plant Physiology, 47 (1971) 649-655.

[16] I. E. Akinci, S. Akinci, and K. Yilmaz, Response of tomato (Solanum lycopersicum L.) to lead toxicity: growth, element uptake, chlorophyll and water content. African Journal of Agriculture Research 5 (2010) 416-423.
[17] C. Wang, Y. Tian, X. Wang, J. Geng, J. Jiang, H. Yu, and C. Wang, Lead-contaminated soil induced oxidative stress, defence response and its indicative biomarkers in roots of Vicia faba seedlings. Ecotoxicology, 19 (2010) 1130-1139.

[18] Y. Ekmekçi, D. Tanyolaç, and B. Ayhan, A crop tolerating oxidative stress induced by excess lead: maize. Acta Physiologiae Plantarum, 31 (2009) 319-330.

[19] C. Sudhakar, L. Syamalabai, and K. Veeranjaneyulu, Lead tolerance of certain legume species grown on lead ore tailings. Agric Ecosyst Environ, 41 (1992) 253-261.

[20] R. Alkhatib, J. Maruthavanan, S. Ghoshroy, R. Steiner, T. Sterling, and R. Creamer, Physiological and ultra structural effects of lead on tobacco. Biologia Plantarum, 56 (2011) 711-716.

[21] Y. Yang, X. Wei, J. Lu, J. You, W. Wang, and R. Shi, Lead-induced phytotoxicity mechanism involved in seed germination and seedling growth of wheat (Triticum aestivum L.). Ecotoxicology and Environmental Safety, 73 (2010) 1982-1987.

[22] S. D. Lane, and E. S. Martin, A histochemical investigation of lead uptake in Raphanus sativus. New Phytology, 79 (1977) 281-286.

[23] R. Przymusinski, M. Spychala, and E. A. Gwozdz, Inorganic lead changes growth polypeptide pattern of lupin roots. Biochemie und Physiolgie Pflanzen, 187 (1991) 51-57.

[24] R. M. Cox, and T. C. Hutchinson, Multiple and co-tolerance in the grass Deschampsia cespitosa: adaptation, pre adaptation and cost. Journal of Plant Nutrition, 3 (1981) 731-741.

[25] S. A. Bharwana, S. Ali, M. A. Farooq, N. Iqbal, F. Abbas, and M. S. A. Ahmad, Alleviation of lead toxicity by silicon is related to elevated photosynthesis, antioxidant enzymes suppressed lead uptake and oxidative stress in cotton. Journal of Bioremediation and Biodegradation, 4 (2013) 1-11.

[26] H. N. Azad, A. H. Shiva, and R. Malekpour, Toxic effects of lead on growth and some biochemical and ionic parameters of sunflower (Helianthus annuus L) seedlings. Current Research Journal Biological Sciences, 3 (2011) 398-403.

[27] S. S. Gill, N. A. Khan, and N. Tuteja, Cadmium at high dose perturbs growth, photosynthesis and nitrogen metabolism while at low dose it up regulates sulfur assimilation and antioxidant machinery in garden cress (Lepidium sativum L.). Plant Science, 182 (2012) 112-120.

[28] S. Zhao, X. Ye, and J. Zheng, Lead-induced changes in plant morphology, cell ultrastructure, growth and yields of tomato. African Journal of Biotechnology, 10 (2011) 10116-10124.

[29] A. J. M. Baker, and P. L. Walker, Ecophysiology of metal uptake by tolerant plants. In: A. J. Shaw (Ed.), Heavy metal tolerance in plants: Evolutionary aspects. CRC Press, Boca Raton, FL, pp. 155-177.

[30] T. C. Broyer, C. M. Johnson, R. E. Paul, Some aspects of lead in plant nutrition. Plant and Soil, 36 (1972) 301-313.

[31] J. Liu, K. Li, J. Xu, Z. Zhang, T. Ma, J. Xang, Q. Zhu, Lead toxicity, uptake and translocation in different rice cultivars. Plant Science, 165 (2004) 793-802.

[32] D. Cargnelutti, L. A. Tabaldi, R. M. Spanevello, G. O. Jucoski, V. Battisti, M. Redin, C. E. Linares, V. L. Dressler, E. M. M. 
Flores, F. T. Nicoloso, V. M. Morsch, and M. R. Schetinger, Mercury toxicity induces oxidative stress in growing cucumber seedlings. Chemosphere, 65 (2006) 999-1006.

[33] H. S. El-Beltagi, and A. A. Mohamed, Changes in non protein thiols, some antioxidant enzymes activity and ultra structural alteration in radish plant (Raphanus sativus L.) grown under lead toxicity. Notulae Botanicae Horti Agrobotanici Cluj-Napoca, 38 (2010) 76-85.

[34] L. Chen, S. Gao, P. Zhu, Y, Liu, T. Hu, and J. Zhang, Comparative study of metal resistance and accumulation of lead and zinc in two poplars. Physiologia Plantarum, 151 (2014) 390-405.

[35] P. Wang, S. Zhang, C. Wang, and J. Lu, Effects of $\mathrm{Pb}$ on the oxidative stress and antioxidant response in a $\mathrm{Pb}$ bioaccumulator plant Vallisneria natans. Ecotoxicology and Environmental Safety, 78 (2012) 28-34.

[36] A. Kumar, M. N. V. Prasad, and O. Sytar, Lead toxicity, defense strategies and associated indicative biomarkers in Talinum triangulare grown hydroponically. Chemosphere, 89 (2012) 1056-1065. 